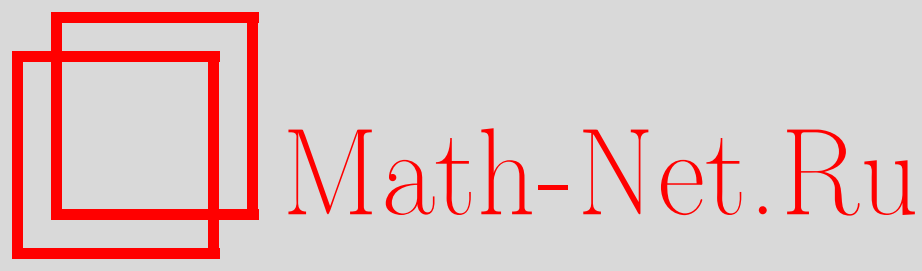

Д. И. Дьяконов, В. Ю. Петров, А. А. Владимиров, Барионные резонансы в релятивистском подходе среднего поля, ТМФ, 2012, том 170, номер 1, 140-160

DOI: https://doi.org/10.4213/tmf6753

Использование Общероссийского математического портала Math-Net.Ru подразумевает, что вы прочитали и согласны с пользовательским соглашением http://www.mathnet.ru/rus/agreement

Параметры загрузки:

IP: 52.90 .164 .192

26 апреля 2023 г., 18:16:06

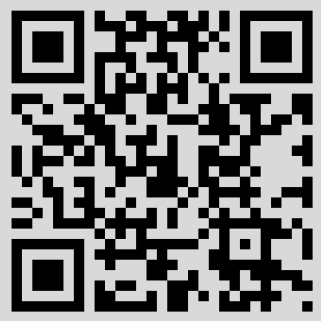




\title{
ФИЗИКА
}

Том 170, № 1

январь, 2012

(C) 2012 г.

Д. И. Дьяконов*†, В. Ю. Петров ${ }^{* \dagger}$, А. А. Владимиров ${ }^{\dagger}$

\section{БАРИОННЫЕ РЕЗОНАНСЫ В РЕЛЯТИВИСТСКОМ ПОДХОДЕ СРЕДНЕГО ПОЛЯ}

\begin{abstract}
Предложен новый подход, согласно которому барионные резонансы можно рассматривать как коллективные возбуждения относительно "внутренних" однокварковых возбуждений в среднем поле определенной симметрии. Такая точка зрения подтверждается в пределе большого числа цветов $N_{\text {c }}$. Хотя в реальном мире $N_{\mathrm{c}}=3$, хорошее согласие с наблюдаемым резонансным спектром получается вплоть до 2 ГэВ. Возможным следствием данной схемы является существование новых экзотических очарованных (и $b$-кварков) барионов, которые могут быть устойчивыми при сильных распадах.
\end{abstract}

Ключевые слова: квантовая хромодинамика, барионы, резонансы, среднее поле.

\section{1. ВВЕДЕНИЕ}

Если число цветов $N_{\text {c }}$ велико, то составляющие барион $N_{\text {c }}$ кварков можно рассматривать в среднем (не флуктуирующем) поле, которое не изменяется при

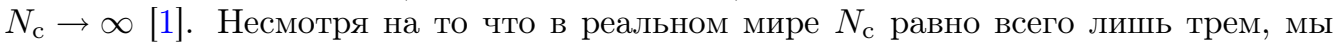
не ожидаем, что барионный спектр будет качественно отличаться от своего предела при больших $N_{\text {c. }}$ Можно надеяться, что если получить четкую картину при больших $N_{\mathrm{c}}$, то ее следы удастся обнаружить и при $N_{\mathrm{c}}=3$.

Преимущество подхода больших $N_{\text {c }}$ состоит в том, что в этом случае барионная физика значительно упрощается, что позволяет полностью учитывать важные релятивистские и теоретико-полевые эффекты, которыми зачастую пренебрегают. Барионы представляют собой не просто три (или $N_{\mathrm{c}}$ ) кварка, они дополнительно содержат кварк-антикварковые пары, что хорошо известно из экспериментов. Теоретически число антикварков в барионах также пропорционально $N_{\text {c }}$ [2], это означает, что антикварки нельзя получить путем добавления одного мезона к бариону: требуется $O\left(N_{\mathrm{c}}\right)$ мезонов для объяснения $O\left(N_{\mathrm{c}}\right)$ антикварков, что в действительности подразумевает классическое мезонное поле.

* Петербургский институт ядерной физики им. Б. П. Константинова, Гатчина, Ленинградская обл., Россия. E-mail: dmitri.diakonov@gmail.com, victorp@thd.pnpi.spb.ru

${ }^{\dagger}$ Institute for Theoretical Physics-II, Ruhr-Universität Bochum, Bochum, Germany. E-mail: vladimirov.aleksey@googlemail.com 
Барионные резонансы могут состоять не только из кварковых возбуждений, как в нерелятивистских кварковых моделях, но и из возбуждений частица-дырка и пе-

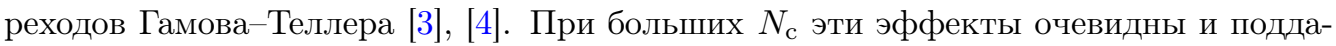
ются анализу, тогда как при $N_{\mathrm{c}}=3$ они представляют собой сложную совокупность явлений, носящую название сильные взаимодействия.

На микроскопическом уровне кварки испытывают только "цветные" взаимодей-

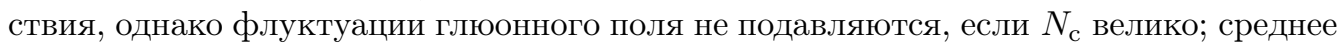
поле может быть только “бесцветным". Пример того, как исходно цветные взаимодействия превращаются с помощью преобразования Фирца во взаимодействия кварков с мезонными полями, дается моделью инстантонной жидкости [5]. Нефлуктуирующий удерживающий мешок представляет собой еще один пример "бесцветного" среднего поля. Более современный пример среднего поля - пятимерное или шестимерное "гравитационное" фоновое поле в АдС/КХД-моделях.

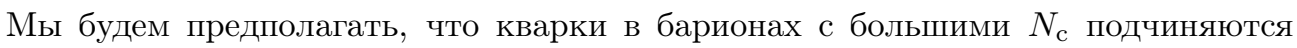
уравнению Дирака в фоновом мезонном поле, поскольку нет причин ожидать, что кварки будут нерелятивистскими, особенно в возбужденных барионах. Все внутренние кварковые дираковские уровни в среднем поле устойчивы при изменении

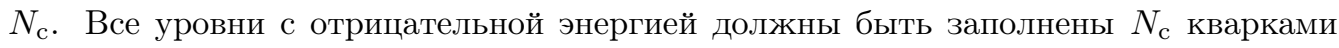
в антисимметричном по цвету состоянии, соответствующем нулевому барионному

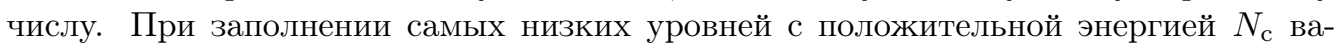
лентными кварками образуется барион. Возбуждение высших кварковых уровней или создание возбуждений частица-дырка порождают барионные резонансы. Барионная масса - величина порядка $O\left(N_{\mathrm{c}}\right)$, а энергия возбуждения - порядка $O(1)$. При возбуждении одного кварка заряд среднего поля равен по порядку величины $O\left(1 / N_{\text {c }}\right)$, и в первом приближении им можно пренебречь.

Более того, если заменить один легкий $(u, d$ или $s)$ кварк в легких барионах на тяжелый $(c, b)$, как в очарованных или $b$-барионах, то изменение среднего поля также будет порядка $O\left(1 / N_{\mathrm{c}}\right)$. Поэтому спектр тяжелых барионов непосредственно связан со спектром легких барионов. Мы покажем, что эти соотношения отвечают реальности.

Наш подход можно проиллюстрировать с помощью модели кирального кваркового солитона [6] или модели кирального мешка [7], однако на самом деле аргументы настоящей работы являются гораздо более общими. Мы утверждаем, что среднее поле в барионах имеет определенную симметрию, а именно, оно спонтанно наруша-

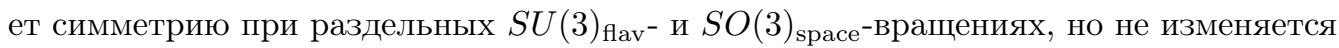

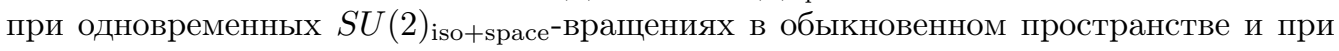
компенсирующем вращении в изопространстве [3], [4].

Отсюда следует, что каждое внутреннее состояние кварка, будь то основное состояние или однокварковое возбуждение в дираковском спектре, порождает зону резонансов, проявляющихся как коллективные вращательные возбуждения данного внутреннего состояния. Квантовые числа этих резонансов, их полное число и расщепления однозначно обусловлены симметрией среднего поля. Если предположить,

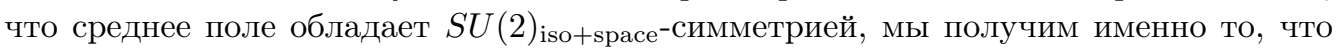
наблюдается в природе. Более того, некоторые соотношения между расщеплениями резонансов, выполненные с высокой точностью, не зависят от динамики, а следуют исключительно из конкретной симметрии среднего поля. 
В настоящей работе мы не будем рассматривать конкретные динамические модели, а сконцентрируемся главным образом на симметрии. Из конкретной динамической модели должен следовать внутренний спектр релятивистского кварка, входящего в состав барионов. Он может получиться из модели почти правильно или совершенно неправильно. Вместо того чтобы вычислять внутренний дираковский спектр кварков из модели, можно получить его из барионного спектра, известного из экспериментов, если интерпретировать барионные резонансы как коллективные возбуждения вблизи основного состояния и вблизи однокварковых переходов. Однако мы покажем, что искомый внутренний спектр кварков можно получить с помощью естественного выбора среднего поля, удовлетворяющего $S U(2)$ iso+space-симметрии.

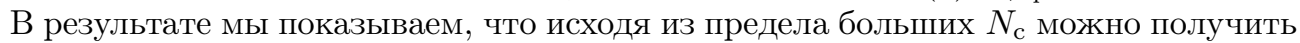
реалистичный спектр барионных резонансов вплоть до 2 ГэВ.

\section{2. СИММЕТРИЯ СРЕДНЕГО ПОЛЯ}

В приближении среднего поля, оправданном при больших $N_{\mathrm{c}}$, будем искать решения уравнения Дирака для состояний одиночного кварка в фоновом среднем поле. В более общем случае фоновое поле взаимодействует с кварками посредством всех пяти ферми-структур. Если среднее поле стационарно во времени, оно приводит к собственному значению уравнения Дирака для $u-, d-, s$-кварков в фоновом поле, $H \psi=E \psi$. При этом гамильтониан Дирака имеет вид

$$
H=\gamma^{0}\left(-i \partial_{i} \gamma^{i}+S(\mathbf{x})+P(\mathbf{x}) i \gamma^{5}+V_{\mu}(\mathbf{x}) \gamma^{\mu}+A_{\mu}(\mathbf{x}) \gamma^{\mu} \gamma^{5}+T_{\mu \nu}(\mathbf{x}) \frac{i}{2}\left[\gamma^{\mu} \gamma^{\nu}\right]\right),
$$

где $S, P, V, A, T$ - соответственно скаляр, псевдоскаляр, вектор, аксиальный вектор и тензор среднего поля; все они являются матрицами по аромату. В действительности одночастичный гамильтониан Дирака (1), вообе говоря, нелокален, однако это не нарушает те симметрии, которые интересуют нас в первую очередь. Мы включили массы токовых и динамически порожденных кварков в скалярный член $S$.

Ключевым моментом является симметрия среднего поля. Мы предполагаем киральный предел для $u$-, $d$-кварков, $m_{u}=m_{d}=0$, что является превосходной ап-

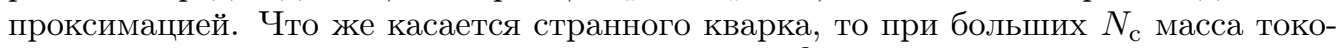
вого странного кварка очень мала, $m_{s}=O\left(1 / N_{\mathrm{c}}^{2}\right)[3]$, поэтому хорошей исходной точкой служит точная $S U(3)$-симметрия по ароматам. Из нее следует, что барионы появляются в вырожденных $S U(3)$-мультиплетах $\mathbf{8}, \mathbf{1 0}, \ldots$; расщепление внутри $S U(3)$-мультиплетов можно определить позже как возмущение по $m_{s}$ (см., например, [8]).

При этом естественно было бы предположить, что среднее поле симметрично по ароматам и сферически-симметрично. Однако нам известно, что барионы сильно взаимодействуют с псевдоскалярными мезонами $\left(g_{\pi N N} \approx 13\right)$. Это означает, что внутри барионов имеется большое псевдоскалярное поле; при больших $N_{\text {c }}$ оно представляет собой классическое среднее поле. Невозможно записать псевдоскалярное поле (которое должно менять знак при инверсии координат), которое было бы совме-

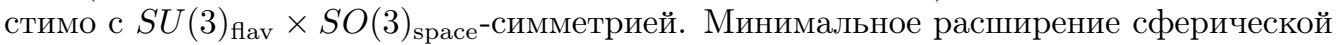
симметрии заключается в том, чтобы записать "ежовый" анзац, "перепутывающий" изотопические и пространственные оси ${ }^{1)}$ :

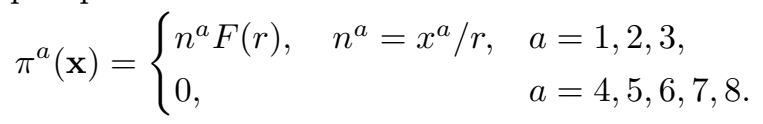

1) А. Хосака сообщил нам, что такой анзац для пионного поля в нуклоне впервые был предложен в 1942 г. в работе [9]; он снова возник в 1961 г. в основополагающих работах [10]. 
Этот анзац нарушает $S U(3)_{\text {flav }}$-симметрию. Более того, он нарушает симметрию при

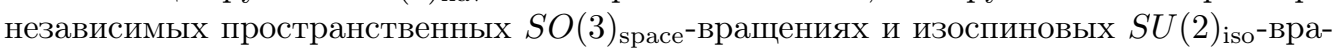
щениях, и только одновременное вращение в обоих пространствах сохраняет симметрию, поскольку вращение в изоспиновом пространстве, к которому относится индекс $a$, можно компенсировать вращением пространственных осей. Из анзаца (2) следует спонтанное (в отличие от явного) нарушение исходной $S U(3)_{\text {flav }} \times$

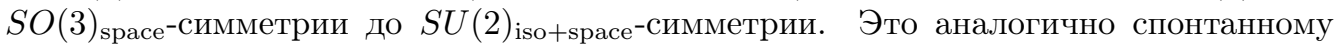
нарушению сферической симметрии эллипсоидальной формой многих ядер; в физике имеется много других примеров, когда исходная симметрия спонтанно нарушается в основном состоянии.

Мы перечислим все возможные структуры для полей $S, P, V, A, T$, согласую-

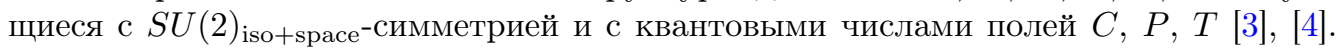
Приведенные ниже поля являются обобщением "ежового" анзаца (2) на мезонные поля с другими квантовыми числами.

Поскольку $S U(3)$-симметрия нарушена, все поля можно разделить на три категории.

1. Изовекторные поля, действующие на $u$-, $d$-кварки:

$$
\text { псевдоскаляр } \quad P^{a}(\mathbf{x})=n^{a} P_{0}(r),
$$

вектор, пространственные компоненты $V_{i}^{a}(\mathbf{x})=\epsilon_{a i k} n_{k} P_{1}(r)$,

аксиальный вектор, пространственные компоненты $A_{i}^{a}(\mathbf{x})=\delta_{a i} P_{2}(r)+$

$$
\begin{aligned}
& +n_{a} n_{i} P_{3}(r), \\
\text { тензор, пространственные компоненты } \quad T_{i j}^{a}(\mathbf{x})= & \epsilon_{a i j} P_{4}(r)+ \\
& +\epsilon_{j} n_{a} n_{b} P_{5}(r) .
\end{aligned}
$$

2. Изоскалярные поля, действующие на $u$-, $d$-кварки:

$$
\begin{aligned}
\text { скаляр } & S(\mathbf{x})=Q_{0}(r), \\
\text { вектор, временна́я компонента } & V_{0}(\mathbf{x})=Q_{1}(r), \\
\text { тензор, смешанные компоненты } & T_{0 i}(\mathbf{x})=n_{i} Q_{2}(r) .
\end{aligned}
$$

3. Изоскалярные поля, действующие на $s$-кварки:

$$
\begin{aligned}
\text { скаляр } & S(\mathbf{x})=R_{0}(r), \\
\text { вектор, временные компоненты } & V_{0}(\mathbf{x})=R_{1}(r), \\
\text { тензор, смешанные компоненты } & T_{0 i}(\mathbf{x})=n_{i} R_{2}(r) .
\end{aligned}
$$

Все остальные поля и компоненты равны нулю, поскольку они не удовлетворя-

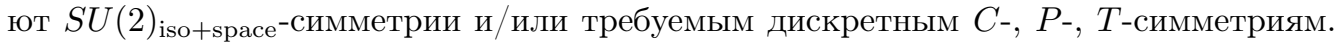
Двенадцать “профильных" функций $P_{0,1,2,3,4,5}, Q_{0,1,2}$ и $R_{0,1,2}$ в конце концов должны быть найдены самосогласованно путем минимизации массы бариона в основном состоянии. Будем называть выражения (3)-(5) "ежовым" анзацем. Из этого анзаца можно получить важные следствия для барионного спектра, даже не зная вида профильных функций. 


\section{3. $u-, d-, s$-КВАРКИ В "ЕЖКОВОМ" ПОЛЕ}

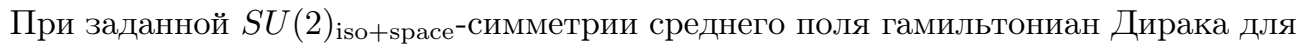
кварков распадается на две составляющие: одна для $s$-кварков, а другая для $u$-, $d$-кварков [3]. Следует подчеркнуть, что энергетические уровни для $u$-, $d$-кварков, с одной стороны, и для $s$-кварков, с другой стороны, полностью различны, даже в киральном пределе $m_{s} \rightarrow 0$.

Энергетические уровни для $s$-кварков классифицируются полуцелыми $J^{P}$, где $P$ - четность при пространственной инверсии, при этом $\mathbf{J}=\mathbf{L}+\mathbf{S}$ - момент импульса кварка; все уровни $(2 J+1)$-кратно вырожденны. Энергетические уровни для $u-$, $d$-кварков классифицируются целыми $K^{P}$, где $\mathbf{K}=\mathbf{T}+\mathbf{J}-$ "большой спин" ( $T$ изоспин), и являются $(2 K+1)$-кратно вырожденными.

Все энергетические уровни, как положительные, так и отрицательные, являются, вероятно, дискретными из-за конфайнмента. Действительно, непрерывный спектр должен соответствовать ситуации, когда кварки свободны на больших расстояниях от центра, что противоречит конфайнменту. Можно моделировать конфайнмент, например, вынуждая эффективные массы кварков линейно возрастать на бесконечности, $S(\mathbf{x}) \rightarrow \sigma r$.

Уравнение Дирака (1) для $s$-кварков в фоновом поле (5) принимает вид системы двух обыкновенных дифференциальных уравнений для двух функций $f(r), g(r)$, зависящих только от расстояния от центра. Система уравнений зависит от (полуцелого) момента импульса рассматриваемого уровня и от его четности. Для уровней с четностью $P=(-1)^{J-1 / 2}$, например для уровней $J^{P}=1 / 2^{+}, 3 / 2^{-}, 5 / 2^{+}, \ldots$, система принимает вид

$$
\begin{aligned}
& E f=-g^{\prime}-\frac{J+3 / 2}{r} g+R_{0} f+R_{1} f+R_{2} g, \\
& E g=f^{\prime}+\frac{-J+1 / 2}{r} f-R_{0} g+R_{1} g+R_{2} f .
\end{aligned}
$$

Чтобы найти энергетический уровень $E$ для $s$-кварка с такими квантовыми числами, нужно решить уравнения (6) с начальными условиями $f(r) \sim r^{J-1 / 2}, g(r) \sim r^{J+1 / 2}$, причем обе функции убывают на бесконечности.

Для уровней с противоположной четностью $P=(-1)^{J+1 / 2}$, например для $J^{P}=$ $1 / 2^{-}, 3 / 2^{+}, 5 / 2^{-}, \ldots$, нужно решить другую систему:

$$
\begin{aligned}
& E f=-g^{\prime}-\frac{-J+1 / 2}{r} g+R_{0} f+R_{1} f+R_{2} g, \\
& E g=f^{\prime}+\frac{J+3 / 2}{r} f-R_{0} g+R_{1} g+R_{2} f .
\end{aligned}
$$

Заметим, что в отсутствие полей $R_{1,2}$ энергетический спектр симметричен относительно одновременных изменений знаков четности и энергии.

Уравнение Дирака для $u$-, $d$-кварков в фоновых полях (3), (4) сложнее: в этом случае мы имеем систему из четырех обыкновенных дифференциальных уравнений. Эти уравнения представляют собой прямые обобщения уравнений Дирака в “ежовом" поле [11], и их можно вывести аналогично тому, как это сделано в указанной работе. 
Система уравнений Дирака для радиальных функций состояний с четностью $(-1)^{K+1}$, а именно $K^{P}=1^{+}, 2^{-}, \ldots$, имеет вид

$$
\begin{aligned}
E f= & -g^{\prime}-\frac{1+K}{r} g+\left(Q_{0}+Q_{1}+P_{2}+P_{4}\right) f+\left(Q_{2}-P_{1}\right) g- \\
& -\frac{P_{0}-P_{1}}{2 K+1}\left(g+b_{K} h\right)+\frac{P_{3}+P_{5}}{2 K+1}\left(f+b_{K} j\right), \\
E g= & f^{\prime}-\frac{K-1}{r} f+\left(Q_{1}-Q_{0}-P_{2}+P_{4}\right) g+\left(Q_{2}-P_{1}\right) f- \\
& -\frac{P_{0}-P_{1}}{2 K+1}\left(f+b_{K} j\right)+\frac{P_{3}-P_{5}+2 P_{2}-2 P_{4}}{2 K+1}\left(g+b_{K} h\right), \\
E h= & j^{\prime}+\frac{2+K}{r} j+\left(Q_{1}-Q_{0}-P_{2}+P_{4}\right) h+\left(Q_{2}-P_{1}\right) j+ \\
& +\frac{P_{0}-P_{1}}{2 K+1}\left(j-b_{K} f\right)-\frac{P_{3}-P_{5}+2 P_{2}-2 P_{4}}{2 K+1}\left(h-b_{K} g\right), \\
E j= & -h^{\prime}+\frac{K}{r} h+\left(Q_{0}+Q_{1}+P_{2}+P_{4}\right) j+\left(Q_{2}-P_{1}\right) h+ \\
& +\frac{P_{0}-P_{1}}{2 K+1}\left(h-b_{K} g\right)-\frac{P_{3}+P_{5}}{2 K+1}\left(j-b_{K} f\right),
\end{aligned}
$$

где $b_{K}=2 \sqrt{K(K+1)}$. Радиальные функции $f, g, h, j$ относятся к парциальным волнам с $L=K-1, K, K, K+1$ соответственно и в начале координат ведут себя как $r^{L}$. Чтобы найти энергетические уровни для заданных $K^{P}$, нужно решить эти уравнения дважды: один раз с начальным условием $f\left(r_{\min }\right) \sim r_{\min }^{K-1}$, полагая при этом все остальные функции равными нулю в начале координат, а второй раз с начальным условием $h\left(r_{\min }\right) \sim r_{\min }^{K}$, полагая все остальные функции равными нулю в начале координат. Затем, численно моделируя эволюцию функций в соответствии с уравнениями (8) до асимптотически большого $r_{\max }$, находим два набора функций $\left(f_{1}, g_{1}, h_{1}, j_{1}\right)$ и $\left(f_{2}, g_{2}, h_{2}, j_{2}\right)$. Энергетические уровни получаем из условий равенства нулю двух (равных) определителей: $f_{1} h_{2}-f_{2} h_{1}=g_{1} j_{2}-g_{2} j_{1}$.

Для состояний с четностью $(-1)^{K}$, а именно $K^{P}=1^{-}, 2^{+}, \ldots$, система уравнений Дирака имеет вид

$$
\begin{aligned}
E f= & -g^{\prime}-\frac{1+K}{r} g+\left(Q_{1}-Q_{0}+P_{2}-P_{4}\right) f-\left(Q_{2}+P_{1}\right) g+ \\
& +\frac{P_{0}+P_{1}}{2 K+1}\left(g+b_{K} h\right)+\frac{P_{3}-P_{5}}{2 K+1}\left(f+b_{K} j\right), \\
E g= & f^{\prime}-\frac{K-1}{r} f+\left(Q_{0}+Q_{1}-P_{2}-P_{4}\right) g-\left(Q_{2}+P_{1}\right) f+ \\
& +\frac{P_{0}+P_{1}}{2 K+1}\left(f+b_{K} j\right)+\frac{P_{3}+P_{5}+2 P_{2}+2 P_{4}}{2 K+1}\left(g+b_{K} h\right), \\
E h= & j^{\prime}+\frac{2+K}{r} j+\left(Q_{0}+Q_{1}-P_{2}-P_{4}\right) h-\left(Q_{2}+P_{1}\right) j- \\
& -\frac{P_{0}+P_{1}}{2 K+1}\left(j-b_{K} f\right)-\frac{P_{3}+P_{5}+2 P_{2}+2 P_{4}}{2 K+1}\left(h-b_{K} g\right), \\
E j= & -h^{\prime}+\frac{K}{r} h+\left(Q_{1}-Q_{0}+P_{2}-P_{4}\right) j-\left(Q_{2}+P_{1}\right) h- \\
& -\frac{P_{0}+P_{1}}{2 K+1}\left(h-b_{K} g\right)-\frac{P_{3}-P_{5}}{2 K+1}\left(j-b_{K} f\right),
\end{aligned}
$$




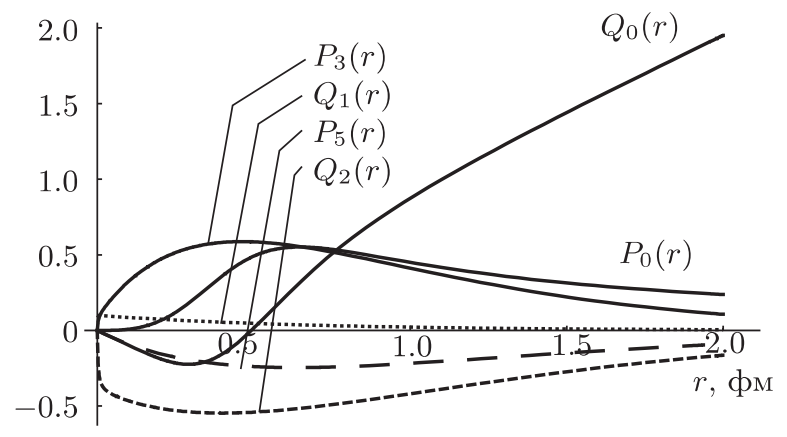

a

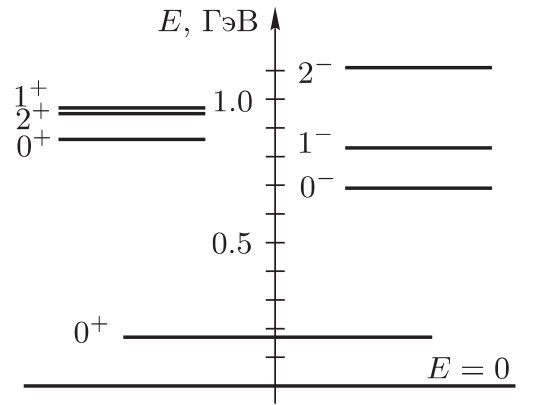

6

Рис. 1. Пример внутренних кварковых уровней с квантовыми числами $K^{P}($ a), порожденных средними полями (б).

где по-прежнему $f \sim r^{K-1}, g \sim r^{K}, h \sim r^{K}, j \sim r^{K+1}$, а уровни находятся точно таким же способом. Поля $Q_{1,2}$ и $P_{0,2,3}$ нарушают симметрию относительно одновременного изменения знаков четности и энергии.

Случай $K=0$ является специальным, поскольку момент импульса ограничивается только одним значением, $J=K+1 / 2=1 / 2$. Это означает, что $g=h=0$, и система уравнений (8) для уровней $K^{P}=0^{-}$редуцируется к двум уравнениям:

$$
\begin{aligned}
& E j=-h^{\prime}+\left(Q_{0}+Q_{1}+P_{2}-P_{3}+P_{4}-P_{5}\right) j+\left(P_{0}-2 P_{1}+Q_{2}\right) h, \\
& E h=j^{\prime}+\frac{2}{r} j+\left(-Q_{0}+Q_{1}-3 P_{2}-P_{3}+3 P_{4}+P_{5}\right) h+\left(P_{0}-2 P_{1}+Q_{2}\right) j,
\end{aligned}
$$

где $h \sim r^{0}, j \sim r^{1}$. Аналогично, чтобы найти уровни $K^{P}=0^{+}$, нужно решить только два уравнения

$$
\begin{aligned}
& E j=-h^{\prime}+\left(-Q_{0}+Q_{1}+P_{2}-P_{3}-P_{4}+P_{5}\right) j-\left(P_{0}+2 P_{1}+Q_{2}\right) h, \\
& E h=j^{\prime}+\frac{2}{r} j+\left(Q_{0}+Q_{1}-3 P_{2}-P_{3}-3 P_{4}-P_{5}\right) h-\left(P_{0}+2 P_{1}+Q_{2}\right) j .
\end{aligned}
$$

На рис. 1 приведен пример кварковых уровней, полученных при "естественном" выборе внешних полей $Q_{0-2}, P_{0-5}$. Мы взяли удерживающее скалярное поле $S(r)=$ $\sigma r$ со стандартным натяжением струны $\sigma=(0.44 \text { ГэВ })^{2}$ и топологическое киральное угловое поле $P(r)=2 \operatorname{arctg}\left(r_{0}^{2} / r^{2}\right)$, так что профильные функции, введенные в (3), (4), - это $Q_{0}(r)=S(r) \cos P(r), P_{0}(r)=S(r) \sin P(r)$; остальные профильные функции экспоненциально убывают на больших расстояниях. Внешние поля показаны на рис. 1a, а получающиеся при различных $K^{P}$ кварковые уровни - на рис. 1б. Как мы увидим, эти или аналогичные уровни определяют массы барионных резонансов.

\section{4. БАРИОНЫ В ОСНОВНОМ СОСТОЯНИИ}

Согласно теории Дирака все уровни с отрищательной энергией, как для $s$-, так и для $u$ - $d$-кварков, должны быть полностью заняты, что отвечает вакууму. Это

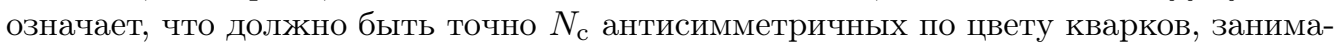
ющих все вырожденные уровни с $J_{3}$ от $-J$ до $J$ или $K_{3}$ от $-K$ до $K$; они образуют

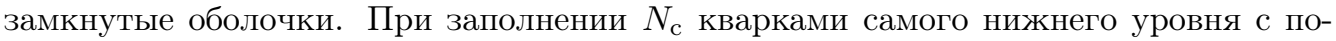
ложительной энергией образуется барион [6], [3] (см. рис. 2). Аналогичная картина наблюдается в киральной модели мешков [7]. 


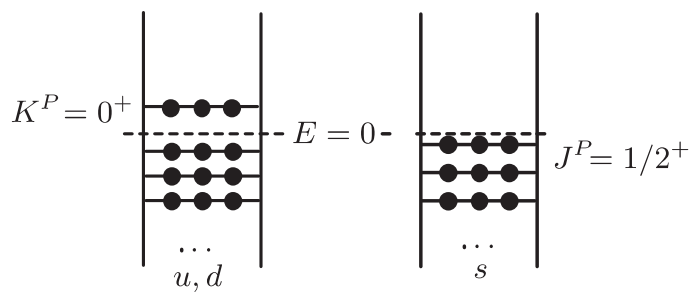

a

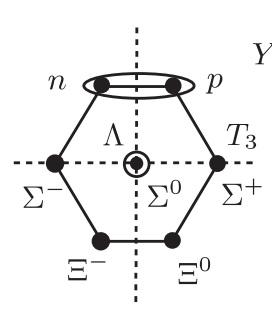

$\left(8,1 / 2^{+}\right)$

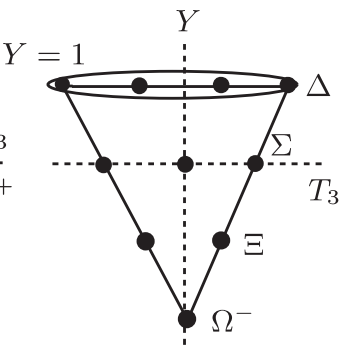

$\left(10,3 / 2^{+}\right)$ б

Рис. 2. Заполнение $u$-, $d$ - и $s$-оболочек для основного состояния бариона (а) и два самых низших барионных мультиплета, получающихся при квантовании вращений такой схемы заполнения (б).

Масса бариона представляет собой совокупную энергию всех заполненных состояний и, будучи функционалом мезонного поля, пропорциональна $N_{\mathrm{c}}$, поскольку все кварковые уровни вырожденны по цвету. Поэтому квантовые флуктуации мезонного поля в барионах подавлены фактором $1 / N_{\mathrm{c}}$, так что среднее поле действительно обоснованно.

Квантовые числа самых легких барионов определяются путем квантования вращений среднего поля, что приводит к конкретным $S U\left(N_{\mathrm{c}}\right)$-мультиплетам, которые редуцируются при $N_{\text {c }}=3$ к октету со спином $1 / 2$ и декуплету со спином $3 / 2$ (см., например, [2]). Условие квантования Виттена для гиперзаряда верхней линии мультиплета, $\widetilde{Y}=N_{\mathrm{c}} / 3$ [12], тривиальным образом следует из того факта, что имеются

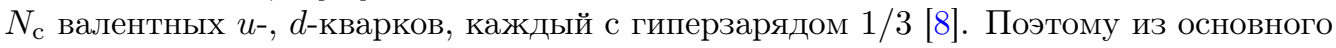
состояния, показанного на рис. 2, действительно следует 56 вращательных состояний. Расщепление между центрами мультиплетов $\left(\mathbf{8}, 1 / 2^{+}\right)$и $\left(\mathbf{1 0}, 3 / 2^{+}\right)$имеет порядок $O\left(1 / N_{\mathrm{c}}\right)$, а расщепление внутри мультиплетов можно найти как возмущение по $m_{s}$ [8].

Самый низкий барионный резонанс, помимо вращательных возбуждений основного состояния, является синглетом $\Lambda\left(1405,1 / 2^{-}\right)$. По-видимому, его можно получить только как возбуждение $s$-кварка, а его квантовые числа должны быть равны $J^{P}=1 / 2^{-}[3]$ (см. переход 1 на рис. 3). Аналогично должен существовать чуть более высокий уровень для $s$-кварка с квантовыми числами $J^{P}=3 / 2^{-}$, соответствующими $\Lambda\left(1520,3 / 2^{-}\right)$(см. переход 2 на рис. 3). Эти два бариона являются единственными известными $S U(3)$-синглетами, и их можно получить только из возбуждений $s$-кварка.

\section{5. ВРАЩАТЕЛЬНЫЕ ЗОНЫ ВБЛИЗИ ВНУТРЕННИХ КВАРКОВЫХ УРОВНЕЙ}

В предположении кирального предела, $m_{u}=m_{d}=m_{s}=0$, исходная симметрия задачи есть $S U(3)_{\text {flav }} \times S O(3)_{\text {space }}$. Эта симметрия, однако, спонтанно нарушается "ежовым" анзацем среднего поля. Соответственно схемы заполнения одночастичных уровней кварка, будь то для основного состояния (рис. 2) или для 


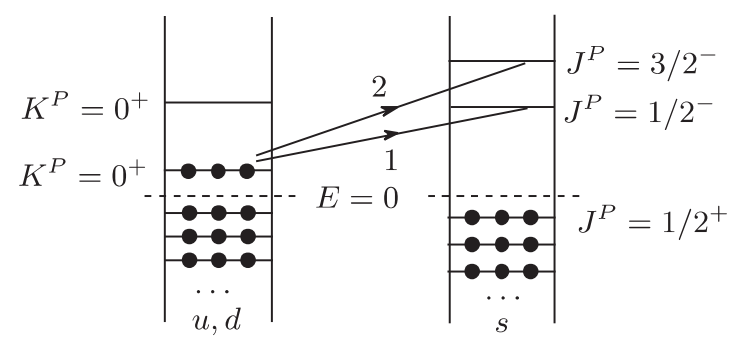

Рис. 3. Однокварковые возбуждения уровней $s$-кварка, соответствующие $S U(3)$-синглетным резонансам, $\Lambda\left(1405,1 / 2^{-}\right)$(переход 1$)$ и $\Lambda\left(1520,3 / 2^{-}\right)$(переход 2).

любых однокварковых возбуждений (см., например, рис. 3), по-разному интерпретируют $u$-, $d$-кварки и $s$-кварки и поэтому нарушают $S U(3)_{\text {flav }}$-симметрию, а так-

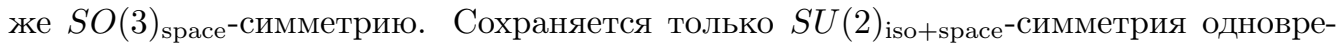
менного изоспинового и компенсирующего пространственного вращений. Исходная

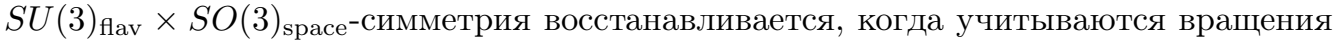
по ароматам и пространственные вращения.

В киральном пределе (который мы предполагаем) произвольное $S U(3)_{\text {flav-враще- }}$ ние среднего поля и, следовательно, того, что мы называем $u-, d-, s$-кварками, не

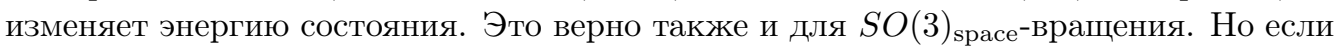

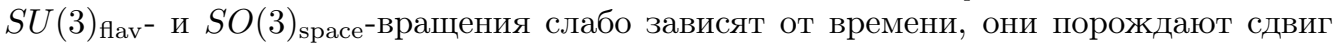
в энергии системы, который называется вращательной энергией.

В соответствии с квантовой механикой эти вращения должны квантоваться. Это приводит к появлению "вращательной зоны" на каждом однокварковом возбужде-

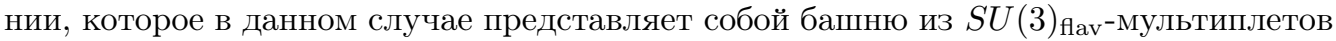
с определенным спином; эти мультиплеты специфичны для каждого однокваркового возбуждения. Расщепление мультиплетов, обусловленное данным однокварковым возбуждением внутри зоны, имеет порядок $O\left(1 / N_{\mathrm{c}}\right)$. В принципе вращательные состояния поднимаются до бесконечности, однако на практике мы ограничиваемся рассмотрением вращательных зон вплоть до поправок по энергии порядка $O(1)$, поскольку столь высокие вращательные состояния могут смешиваться с другими однокварковыми возбуждениями, что требует более тщательного анализа.

Ниже мы кратко остановимся на квантовании $S U(3)_{\text {flav- }}$ и $S O(3)_{\text {space-вращений }}$ вблизи однокваркового возбуждения из основного состояния с $K^{P}=0^{+}$до уровня с некоторым достаточно высоким спином $K$. Насколько нам известно, такое квантование ранее не рассматривалось, и оно представляет определенный интерес.

Пусть $R(t)$ - $S U(3)$-матрица медленных вращений в пространстве ароматов, а $S(t)$ - $S U(2)$-матрица медленных пространственных (и спиновых) вращений. Введем угловые скорости $\widetilde{\Omega}^{a}=-i \operatorname{Tr}\left(R^{\dagger} \dot{R} \lambda^{a}\right)$ и $\widetilde{\omega}^{i}=-i \operatorname{Tr}\left(S^{\dagger} \dot{S} \sigma^{i}\right)$, где $\lambda^{a}$ и $\sigma^{i}-$ восемь матриц Гелл-Манна и три матрицы Паули соответственно. Вращательный лагранжиан имеет вид (см., например, [2])

$$
\mathcal{L}_{\text {rot }}^{(0)}=\frac{I_{1}}{2} \sum_{a=1}^{3}\left(\widetilde{\Omega}^{a}-\widetilde{\omega}^{a}\right)^{2}+\frac{I_{2}}{2} \sum_{a=4}^{7}\left(\widetilde{\Omega}^{a}\right)^{2}-\frac{N_{\mathrm{c}}}{2 \sqrt{3}} \widetilde{\Omega}^{8},
$$


где $I_{1,2}=O\left(N_{\mathrm{c}}\right)$ - два момента инерции. Это хорошо известный вращательный лагранжиан для основного состояния, а также и для вращений вблизи однокварковых возбуждений $u$-, $d$-кварков до уровней с $K^{P}=0^{ \pm}$.

При вращениях вблизи однокварковых возбуждений до уровней с $K \neq 0$ любой четности к вращательному лагранжиану нужно добавить слагаемые, линейные по угловым скоростям. Здесь важно, что уровень $u$-, $d$-кварков при $K \neq 0$ является вырожденной смесью $2 K+1$ состояний с различными проекциями $K_{3},-K \leqslant K_{3} \leqslant K$, характеризующимися волновыми функциями $\chi_{K_{3}}$. При $R$ - или $S$-вращении фонового поля вырождение состояний с различными значениями $K_{3}$ снято, и мы находим дополнительный однокварковый вклад во вращательный лагранжиан:

$$
\begin{aligned}
\mathcal{L}_{\text {rot }}^{(1)} & =\chi_{K_{3}}^{\dagger} i \frac{\partial}{\partial t} \chi_{K_{3}}+\widetilde{\boldsymbol{\Omega}} \cdot \mathbf{T}^{(1)}+\widetilde{\boldsymbol{\omega}} \cdot \mathbf{J}^{(1)}, \\
\mathbf{T}^{(1)} & =\sum_{K_{3}, K_{3}^{\prime}} \chi_{K_{3}^{\prime}}^{\dagger} \chi_{K_{3}}\left\langle K K_{3} J L|\mathbf{T}| K K_{3}^{\prime} J L\right\rangle, \\
\mathbf{J}^{(1)} & =\sum_{K_{3}, K_{3}^{\prime}} \chi_{K_{3}^{\prime}}^{\dagger} \chi_{K_{3}}\left\langle K K_{3} J L|\mathbf{J}| K K_{3}^{\prime} J L\right\rangle,
\end{aligned}
$$

где $\mathbf{J}^{(1)}, \mathbf{T}^{(1)}$ - соответственно момент импульса и изоспин данного состояния кварка $\left|K K_{3} J L\right\rangle$. Очевидно, они удовлетворяют соотношению $\mathbf{J}^{(1)}+\mathbf{T}^{(1)}=\mathbf{K}$, где $\mathbf{K}^{2}=$ $K(K+1)$, а $K$ - большой спин возбужденного уровня. Полный вращательный лагранжиан представляет собой сумму

$$
\mathcal{L}_{\text {rot }}=\mathcal{L}_{\text {rot }}^{(0)}+\mathcal{L}_{\text {rot }}^{(1)}
$$

Для квантования вращений вблизи однокварковых возбуждений введем канонически сопряженные импульсы

$$
\begin{aligned}
\widetilde{\mathbf{T}} & =\frac{\partial \mathcal{L}}{\partial \widetilde{\boldsymbol{\Omega}}}=I_{1}(\widetilde{\boldsymbol{\Omega}}-\widetilde{\boldsymbol{\omega}})+\mathbf{T}^{(1)}, \quad \tilde{\mathbf{J}}=\frac{\partial \mathcal{L}}{\partial \widetilde{\boldsymbol{\omega}}}=-I_{1}(\widetilde{\boldsymbol{\Omega}}-\widetilde{\boldsymbol{\omega}})+\mathbf{J}^{(1)}, \quad \widetilde{\mathbf{T}}+\widetilde{\mathbf{J}}=\mathbf{K}, \\
\widetilde{T}^{a} & =I_{2} \widetilde{\Omega}^{a}, \quad a=4,5,6,7, \quad \widetilde{T}^{8}=-\frac{N_{\mathrm{c}}}{3},
\end{aligned}
$$

и найдем вращательный гамильтониан

$$
\mathcal{H}_{\text {rot }}=\widetilde{T}^{a} \widetilde{\Omega}^{a}+\widetilde{J}^{i} \widetilde{\omega}^{i}-\mathcal{L}_{\text {rot }}=\frac{1}{2 I_{1}} \sum_{a=1}^{3}\left(\widetilde{\mathbf{T}}-\mathbf{T}^{(1)}\right)^{2}+\frac{1}{2 I_{2}} \sum_{a=4}^{7}\left(\widetilde{T}^{a}\right)^{2},
$$

который нужно проквантовать, используя стандартные коммутационные соотношения для $\widetilde{T}^{a}$ и $\widetilde{J}^{i}$, но с условием $\widetilde{\mathbf{T}}+\tilde{\mathbf{J}}=\mathbf{K}$, при этом гиперзаряд равен $\widetilde{Y}=$ $-(2 / \sqrt{3}) \widetilde{T}^{8}=N_{\mathrm{c}} / 3$.

Важное обстоятельство состоит в том, что матричные элементы однокварковых операторов $\mathbf{T}^{(1)}, \mathbf{J}^{(1)}$ пропорциональны $\mathbf{K}$ :

$$
\mathbf{T}^{(1)}=\frac{K+1-(2 K+1) c_{K}}{K(K+1)} \mathbf{K}=\frac{K+1-(2 K+1) c_{K}}{K(K+1)}(\tilde{\mathbf{J}}+\widetilde{\mathbf{T}}),
$$


где коэффициент $c_{K}$ связан с нормировкой радиальных функций уровня $K$ :

$$
c_{K}=\int d r r^{2}\left(h^{2}+j^{2}\right) / \int d r r^{2}\left(f^{2}+g^{2}+h^{2}+j^{2}\right),
$$

и $\tilde{\mathbf{J}}^{2}=\mathbf{J}^{2}=J(J+1)$, где $J$ - полный угловой момент состояния. Это позволяет найти собственные значения оператора

$$
\begin{aligned}
\left(\widetilde{\mathbf{T}}-\mathbf{T}^{(1)}\right)^{2} & =a_{K} J(J+1)+\left(1-a_{K}\right) \widetilde{T}(\widetilde{T}+1)-a_{K}\left(1-a_{K}\right) K(K+1), \\
a_{K} & =\frac{1-c_{K}}{K}-\frac{c_{K}}{K+1} .
\end{aligned}
$$

Второй член в гамильтониане (15) можно записать в следующем виде:

$$
\sum_{a=4}^{7}\left(\widetilde{T}^{a}\right)^{2}=\sum_{a=1}^{8}\left(\widetilde{T}^{a}\right)^{2}-\sum_{a=1}^{3}\left(\widetilde{T}^{a}\right)^{2}-\left(\widetilde{T}^{8}\right)^{2}=C_{2}-\widetilde{T}(\widetilde{T}+1)-\frac{3}{4} \widetilde{Y}^{2},
$$

где $C_{2}=\left(p^{2}+p q+q^{2}+3 p+3 q\right) / 3$ - квадратичный оператор Казимира для $S U(3)$-мультиплета, характеризующегося числами $p, q$ из таблицы Юнга. Для прототипа октета при больших $N_{\text {c }}$ берем [2] $p=1, q=\left(N_{\mathrm{c}}-1\right) / 2$ и $\widetilde{T}=1 / 2, \widetilde{Y}=N_{\mathrm{c}} / 3$, тогда как для прототипа декуплета берем $p=3, q=\left(N_{\mathrm{c}}-3\right) / 2, \widetilde{T}=3 / 2, \widetilde{Y}=N_{\mathrm{c}} / 3$. В обоих случаях второй член в гамильтониане (15) соответствует постоянному сдвигу энергии:

$$
\frac{1}{2 I_{2}} \sum_{a=4}^{7}\left(\widetilde{T}^{a}\right)^{2}=\frac{N_{\mathrm{c}}}{4 I_{2}}=O(1)
$$

Таким образом, массы центров октетов и декуплетов как вращательных возбуждений вблизи однокваркового перехода $u$-, $d$-кварков с уровня основного состояния $0^{+}$на возбужденный уровень $K^{P}$ определяются уравнением

$$
\begin{aligned}
\mathcal{M}= & \mathcal{M}_{0}+\frac{N_{\mathrm{c}}}{4 I_{2}}+\Delta \mathcal{E}\left(0^{+} \rightarrow K^{P}\right)+ \\
& +\frac{a_{K} J(J+1)+\left(1-a_{K}\right) \widetilde{T}(\widetilde{T}+1)-a_{K}\left(1-a_{K}\right) K(K+1)}{2 I_{1}} .
\end{aligned}
$$

Возьмем $\widetilde{T}=1 / 2$ и $\widetilde{T}=3 / 2$ для октета и декуплета соответственно, а возможные спины $J=\tilde{J}$ мультиплетов найдем из закона сложения векторов $\tilde{\mathbf{J}}+\widetilde{\mathbf{T}}=\mathbf{K}$. Например, если взять однокварковое возбуждение из основного состояния $0^{+}$на возбужденный уровень $1^{ \pm}$, соответствующая вращательная зона будет состоять из октетов со спинами $J=1 / 2$ и $J=3 / 2$ и декуплетов со спинами $J=1 / 2,3 / 2,5 / 2$. Массы центров этих мультиплетов определяются уравнением (17), коэффициент $a_{K=1}$

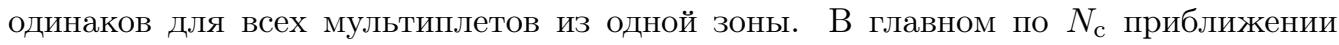
моменты инерции $I_{1,2}$ одинаковы для всех вращательных зон вблизи всех однокварковых возбуждений. 
Для вращательных возбуждений вблизи основного состояния $0^{+}$(или вблизи однокваркового перехода $0^{+} \rightarrow 0^{ \pm}$) требуются только два мультиплета: октет со спином $J=\widetilde{T}=1 / 2$ и декуплет со спином $J=\widetilde{T}=3 / 2$, и уравнение (17) редуцируется к хорошо известному уравнению для расщеплений между их центрами:

$$
\mathcal{M}=\mathcal{M}_{0}+\frac{N_{\mathrm{c}}}{4 I_{2}}+\Delta \mathcal{E}\left(0^{+} \rightarrow 0^{ \pm}\right)+\frac{J(J+1)}{2 I_{1}} .
$$

\section{6. СРАВНЕНИЕ С ЭКСПЕРИМЕНТАЛЬНЫМ СПЕКТРОМ}

Теперь рассмотрим экспериментальный спектр резонансов для легких барионов с массой до 2 ГэВ и попытаемся понять, где находятся вращательные зоны вблизи однокварковых возбуждений из основного состояния во внутренние кварковые уровни с $K^{P}=0^{ \pm}, 1^{ \pm}, 2^{ \pm}$. Мы будем интерпретировать входящие в уравнение (17) величины как свободные параметры, которые нужно подогнать к известным массам, хотя, в принципе, их можно вычислить, если известно (самосогласованное) среднее поле. Тем не менее имеется гораздо больше резонансов, чем свободных параметров, поэтому вращательные зоны в сильной степени запрещены уравнением (17). Как мы увидим, экспериментальные массы удовлетворяют этим ограничениям, несмотря на то что в реальном мире $N_{\mathrm{c}}=3$.

Поскольку до сих пор мы не учитывали расщепление внутри $S U(3)$-мультиплетов, обусловленное ненулевым $m_{s}$, уравнение (17) описывает массу центра мультиплета. Для октета центр определяется как $\mathcal{M}_{8}=\left(2 m_{N}+2 m_{\Xi}+3 m_{\Sigma}+m_{\Lambda}\right) / 8$, а для декуплета - как $\mathcal{M}_{10}=\left(4 m_{\Delta}+3 m_{\Sigma^{*}}+2 m_{\Xi^{*}}+m_{\Omega}\right) / 10 \approx m_{\Sigma^{*}}$. Мы возьмем феноменологические значения для $\mathcal{M}_{8}, \mathcal{M}_{10}$ из работы [13], авторы которой недавно проанализировали барионные мультиплеты вплоть до 2 ГэВ.

6.1. Ложные состояния. При сравнении предсказаний, полученных с помощью метода среднего поля (справедливого при $N_{\text {c }} \rightarrow \infty$ ), с экспериментальными данными нужно учитывать, что некоторые вращательные состояния на самом деле являются ложными, поскольку они представляют собой артефакты, получающиеся в приближении среднего поля, где пространственная волновая функция равна произведению одночастичных волновых функций. При учете усреднения по центру масс (эффект порядка $O\left(1 / N_{\mathrm{c}}\right)$ ) барионные волновые функции зависят только от разностей кварковых координат, что для некоторых состояний может противоречить принципу Паули. Этот эффект давно известен как в ядерной физике [14], так и в нерелятивистской кварковой модели [15]. Простейший способ отождествить ложные состояния состоит в том, чтобы непрерывно деформировать среднее поле к потенциалу нерелятивистского осциллятора, для которого волновые функции явно известны. Если в таком пределе некоторое состояние отсутствует, оно не может появиться вследствие непрерывной деформации. Независимый способ выявления ложных состояний состоит в том, чтобы деформировать данную задачу до точно решаемой $(0+1)$-мерной модели четырехфермионного взаимодействия [16], где также возможно приближение при больших $N_{\mathrm{c}}$, в котором обнаруживаются дополнительные состояния. В секторе с положительной четностью ложным является состояние 
$\left(\mathbf{1 0}, 1 / 2^{+}\right)$, возникающее из вращательной зоны вблизи перехода $\left(0^{+} \rightarrow 2^{+}\right)$. Такое состояние возникает также из перехода $\left(0^{+} \rightarrow 1^{+}\right)$, однако тогда оно является разрешенным.

В секторе с отрицательной четностью имеется больше ложных состояний: мультиплеты $\left(\mathbf{1 0}, 5 / 2^{-}\right)$и $\left(\mathbf{1 0}, 7 / 2^{-}\right)$, обусловленные переходом $\left(0^{+} \rightarrow 2^{-}\right)$, являются ложными, два из трех мультиплетов $\left(\mathbf{1 0}, 3 / 2^{-}\right)$, возникающих из переходов $\left(0^{+} \rightarrow\right.$ $\left.0^{-}, 1^{-}, 2^{-}\right)$, являются ложными, и один из двух мультиплетов $\left(\mathbf{1 0}, 1 / 2^{-}\right)$, возникающий из переходов $\left(0^{+} \rightarrow 1^{-}, 2^{-}\right)$, также является ложным. Ложные вращательные состояния не нужно учитывать при сравнении с экспериментальными данными.

6.2. Резонансы с положительной четностью. Два самых нижних мультиплета, $\left(\mathbf{8}, 1 / 2^{+}, 1149\right)$ и $\left(\mathbf{1 0}, 3 / 2^{+}, 1382\right)$ (последнее число в скобках соответствует центру мультиплета), образуют вращательную зону вблизи основного состояния заполненной схемы, показанной на рис. 2. Подгоняя эти массы с помощью уравнения (18), найдем $\mathcal{M}_{0}+3 /\left(4 I_{2}\right)=1091 \mathrm{MэB,} 1 / I_{1}=155$ МэВ.

Кроме этих самых нижних мультиплетов имеется другая низколежащая пара с теми же квантовыми числами, $\left(\mathbf{8}, 1 / 2^{+}, 1605\right)$ и $\left(\mathbf{1 0}, 3 / 2^{+}, 1732\right)$. Другие мультиплеты с положительной четностью в основном лежат выше. Поэтому для объяснения этой пары требуется переход $0^{+} \rightarrow 0^{+}$. Из подгонки масс находим, что второй внутренний кварковый уровень с $K^{P}=0^{+}$должен быть на 483 МэВ выше, чем уровень $0^{+}$основного состояния, $\Delta \mathcal{E}\left(0^{+} \rightarrow 0^{+}\right)=483$ МэВ. Момент инерции оказывается значительно больше, чем для мультиплетов в основном состоянии, $1 / I_{1}=85 \mathrm{M \ni В.}$ Хотя различие имеет порядок $O\left(1 / N_{\mathrm{c}}\right)$, его можно увеличить, если радиально возбужденный уровень $0^{+}$имеет намного больший эффективный радиус.

Хорошо известная группа из пяти мультиплетов $\left(\mathbf{8}, 3 / 2^{+}, 1865\right),\left(\mathbf{8}, 5 / 2^{+}, 1873\right)$, $\left(\mathbf{1 0}, 3 / 2^{+}, 2087\right),\left(\mathbf{1 0}, 5 / 2^{+}, 2031\right),\left(\mathbf{1 0}, 7 / 2^{+}, 2038\right)$ является кандидатом для вращательной зоны $0^{+} \rightarrow 2^{+}$. Действительно, этот набор в точности повторяет содержание вращательной зоны для данного перехода (ложный мультиплет $\left(\mathbf{1 0 , 1 / 2 ^ { + } )}\right.$ мы исключаем), а подгонка масс согласно уравнению (17) дает небольшое значение $\sqrt{\chi^{2}}=15$ МэВ. Следует, тем не менее, иметь в виду, что не все члены всех мультиплетов хорошо установлены [13], а те, которые установлены, имеют неопределенность экспериментальных значений массы. Это означает, что экспериментальные значения массы для центров мультиплетов известны в лучшем случае с точностью 20-40 МэВ. С помощью подгонки находим $1 / I_{1}=122 \mathrm{MэB}, \Delta \mathcal{E}\left(0^{+} \rightarrow 2^{+}\right)=715$ МэВ. Поэтому внутренний уровень $2^{+}$должен быть выше, чем уровень $0^{+}$.

Единственный относительно хорошо установленный мультиплет, который остается в области ниже 2 ГэВ, - это мультиплет $\left(\mathbf{8}, 1 / 2^{+}, 1846\right)$. Это наводит на мысль, что он может возникать из вращательной зоны вблизи перехода $0^{+} \rightarrow 1^{+}$, однако об остальных частях зоны известно мало. Если рассмотреть остающиеся нестранные барионы, то можно найти $N\left(1 / 2^{+}, 1710^{* * *}\right), N\left(1 / 2^{+}, 1900^{* *}\right), \Delta\left(1 / 2^{+}, 1910^{* * *}\right)$ и $\Delta\left(5 / 2^{+}, 2000^{* *}\right)$, при этом отсутствует $\Delta\left(3 / 2^{+}\right)$. Квантовые числа и массы этих предлагаемых резонансов довольно хорошо согласуются с гипотезой о том, что они возникают как вращательная зона вблизи перехода $0^{+} \rightarrow 1^{+}$, однако их более низкий экспериментальный статус не позволяет сделать определенное заключение. Внутренний уровень $1^{+}$должен быть приблизительно на 70 МэВ выше, чем кварковый уровень $2^{+}$. 
ТАБлицА 1. Интерпретация всех барионных резонансов ниже 2 ГэВ как вращательных возбуждений поверх внутренних кварковых состояний

\begin{tabular}{|c|c|}
\hline Внутренние кварковые уровни & $\begin{array}{c}\text { Мультиплеты, являющиеся вращательными } \\
\text { состояниями вблизи этого перехода }\end{array}$ \\
\hline$K^{P}=0^{+}, \quad$ основное состояние & $\left(\mathbf{8}, 1 / 2^{+}, 1149\right), \quad\left(\mathbf{1 0}, 3 / 2^{+}, 1382\right)$ \\
\hline $0^{+} \rightarrow 0^{+}, \quad 483 \mathrm{MэB}$ & $\left(\mathbf{8}, 1 / 2^{+}, 1605\right), \quad\left(\mathbf{1 0}, 3 / 2^{+}, 1732\right)$ \\
\hline $0^{+} \rightarrow 2^{+}, \quad 715 \mathrm{MэB}$ & $\begin{array}{c}\left(\mathbf{8}, 3 / 2^{+}, 1865\right), \quad\left(\mathbf{8}, 5 / 2^{+}, 1873\right), \quad\left(\mathbf{1 0}, 3 / 2^{+}, 2087\right), \\
\left(\mathbf{1 0}, 5 / 2^{+}, 2031\right), \quad\left(\mathbf{1 0}, 7 / 2^{+}, 2038\right)\end{array}$ \\
\hline $0^{+} \rightarrow 1^{+}, \quad \sim 780 \mathrm{M \ni B}$ & $\begin{array}{c}N\left(1 / 2^{+}, 1710\right), \quad N\left(3 / 2^{+}, 1900\right), \quad \Delta\left(1 / 2^{+}, 1910\right) \\
\Delta\left(3 / 2^{+}, \sim 1945\right) ?, \quad \Delta\left(5 / 2^{+}, 2000\right)\end{array}$ \\
\hline $0^{+} \rightarrow 0^{-}, \quad 458 \mathrm{M \ni B}$ & $\left(\mathbf{8}, 1 / 2^{-}, 1609\right), \quad\left(\mathbf{1 0}, 3 / 2^{-}, 1850\right)$ \\
\hline $0^{+} \rightarrow 1^{-}, \quad 586 \mathrm{M \ni B}$ & $\left(\mathbf{8}, 1 / 2^{-}, 1710\right), \quad\left(\mathbf{8}, 3 / 2^{-}, 1895\right), \quad\left(\mathbf{1 0}, 1 / 2^{-}, 1758\right)$ \\
\hline $0^{+} \rightarrow 2^{-}, \quad 774 \mathrm{MэB}$ & $\left(\mathbf{8}, 3 / 2^{-}, 1673\right), \quad\left(\mathbf{8}, 5 / 2^{-}, 1801\right)$ \\
\hline
\end{tabular}

6.3. Резонансы с отрицательной четностью. Этот случай аналогичен случаю сектора с положительной четностью: нам нужны внутренние кварковые уровни с $K^{P}=0^{-}, 1^{-}, 2^{-}$для объяснения резонансов как принадлежащих к вращательной зоне вблизи этих переходов. Поскольку несколько вращательных состояний в секторе с отрицательной четностью являются ложными, можно ожидать, что найдутся следующие мультиплеты, обусловленные этими переходами: $\left(\mathbf{8}, 1 / 2^{-}\right) \times 2$, $\left(\mathbf{8}, 3 / 2^{-}\right) \times 2,\left(\mathbf{8}, 5 / 2^{-}\right),\left(\mathbf{1 0}, 1 / 2^{-}\right),\left(\mathbf{1 0}, 3 / 2^{-}\right)$. Это в точности наблюдаемые мультиплеты.

Мультиплеты $\left(\mathbf{8}, 1 / 2^{-}, 1609\right)$ и $\left(\mathbf{1 0}, 3 / 2^{-}, 1850\right)$ образуют вращательную зону перехода $0^{+} \rightarrow 0^{-}$, где $1 / I_{1}=161 \mathrm{MэB,} \Delta \mathcal{E}\left(0^{+} \rightarrow 0^{-}\right)=458 \mathrm{MэВ.} \mathrm{Сравнивая} \mathrm{эти}$ результаты с энергией возбуждения уровня $0^{+}$, равной $483 \mathrm{MэB}$, заключаем, что самое низкое внутреннее возбуждение - это возбуждение $0^{-}$, что выглядит вполне разумным.

Мультиплеты $\left(\mathbf{8}, 1 / 2^{-}, 1710\right),\left(\mathbf{8}, 3 / 2^{-}, 1895\right)$ и $\left(\mathbf{1 0}, 1 / 2^{-}, 1758\right)$ образуют вращательную зону перехода $0^{+} \rightarrow 1^{-}$, где $1 / I_{1}=155 \mathrm{MэB}, \Delta \mathcal{E}\left(0^{+} \rightarrow 0^{-}\right)=586$ МэВ.

Наконец, мультиплеты $\left(8,3 / 2^{-}, 1673\right)$ и $\left(8,5 / 2^{-}, 1801\right)$ являются единственными мультиплетами, принадлежащими вращательной зоне вблизи перехода $0^{+} \rightarrow 2^{-}$. Предполагая $1 / I_{1}=155 \mathrm{MэB,} \mathrm{находим} \Delta \mathcal{E}\left(0^{+} \rightarrow 2^{-}\right)=774 \mathrm{MэB}$.

Таким образом, все барионы с положительной и отрицательной четностью в области 2 ГэВ и ниже можно интерпретировать в рамках нашей схемы, предполагая, что все они возникают как вращательные возбуждения вблизи переходов $0^{+} \rightarrow$ $0^{+}, 1^{+}, 2^{+}$и $0^{+} \rightarrow 0^{-}, 1^{-}, 2^{-}$(см. табл. 1 ). Все резонансы удается объяснить, однако появляется дополнительное состояние $\Delta\left(3 / 2^{+}, \sim 1945\right)$, возникающее из перехода $0^{+} \rightarrow 1^{+}$, который пока не наблюдался, а потому это состояние является лишь предсказанием. 


\section{7. $s$-КВАРКИ}

Как было подчеркнуто в разделе $3, s$-кварки находятся в совершенно другом внешнем поле, чем $u$-, $d$-кварки, даже в киральном пределе. Только удерживающие силы, которые мы моделируем линейно возрастающим скалярным полем, одинаковы для всех кварков. Два возбужденных уровня для $s$-кварков показаны на рис. 3 : они необходимы для объяснения синглетных резонансов $\Lambda\left(1 / 2^{-}, 1405\right)$ и $\Lambda\left(3 / 2^{-}, 1520\right)$. Ниже 2 ГэВ других $\Lambda$-синглетов не известно, поэтому в этой области не должно быть внутренних $s$-кварковых уровней с положительной или отрицательной четностью.

Интригующим является вопрос, где находится высший заполненный уровень $s$ кварков. Предположительно, это должен быть уровень с квантовым числом $J^{P}=$ $1 / 2^{+}$, поскольку он обладает максимальной симметрией. При этом могут существовать однокварковые возбуждения с этого уровня как на возбужденные уровни $1 / 2^{-}$ и $3 / 2^{-} s$-кварка, так и на возбужденные уровни $0^{+}, 0^{-}, \ldots u$-, $d$-кварков (см. рис. 4). Переходы первого типа порождают вращательную зону, состоящую из мультиплетов $\left(\mathbf{8}, 1 / 2^{-}\right) \times 2,\left(\mathbf{8}, 1 / 2^{-}\right),\left(\mathbf{1 0}, 1 / 2^{-}\right),\left(\mathbf{1 0}, 3 / 2^{-}\right) \times 2$ и $\left(\mathbf{1 0}, 5 / 2^{-}\right)$[4]. Переходы второго типа, называющиеся согласно принятой в ядерной физике терминологии переходами Гамова-Теллера, порождают экзотические антидекуплеты ( $\left.\overline{\mathbf{1 0}}, 1 / 2^{+}\right)$и др. [3], [4].

Оказывается трудно, если не невозможно, сместить самый высокий заполненный уровень $1 / 2^{+} s$-кварков, который должен удовлетворять уравнениям (6) в "реалистичном" среднем поле, большем $\sim 700 \mathrm{MэВ,} \mathrm{ниже} \mathrm{первого} \mathrm{возбужденного} \mathrm{уровня}$ $1 / 2^{-}$. Поэтому резонансы с отрицательной четностью, порожденные переходом 1 на рис. 4 , должны проявлять себя в спектре ниже 2 ГэВ. Заметим, что такие резонансы будут иметь основательную 5-кварковую компоненту $u(d) u(d) u(d) s \bar{s}$, поскольку они требуют, чтобы $s$-кварк выталкивался с заполненного уровня и помещался на возбужденный. Вероятно, в реальном мире резонансы представляют собой некоторую смесь этих возбуждений с $u$-, $d$-возбуждениями, описанными в разделе 6 . Желательно, чтобы это свойство выполнялось, поскольку, например, хорошо известный резонанс $N\left(1 / 2^{-}, 1535\right)$ испытывает сильное взаимодействие с $\eta$-мезоном [17], которое не объяснимо в противном случае. Переход Гамова-Теллера естественным образом объясняет экзотический $\Theta^{+}$-резонанс [18] в точности в том месте, где его существование предсказывается на основании результатов ряда экспериментов [3], [4].

\section{8. ОЧАРОВАННЫЕ И $b$-БАРИОНЫ}

Если один из легких кварков в легком барионе заменить на тяжелый $b$ - или

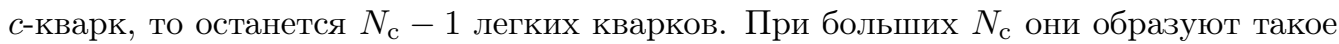
же среднее поле, что и в легких барионах, с той же последовательностью дираков-

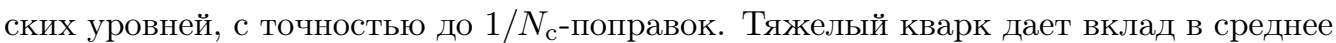
$S U(3)_{\text {flav }}$-симметричное поле, но это также $1 / N_{\mathrm{c}}$-поправка. Это означает, что при больших $N_{\text {с }}$ можно предсказывать спектр $Q q \ldots q$ (и $\left.Q q \ldots q q \bar{q}\right)$ барионов исходя из спектра легких барионов. При $N_{\text {c }}=3$ не ожидается качественного отличия от предела $N_{\mathrm{c}} \rightarrow \infty$, хотя $1 / N_{\mathrm{c}}$-поправки нужно иметь в виду. Рассмотрим тяжелый кварк как нерелятивистскую частицу со спином $J_{\mathrm{h}}=1 / 2$. Заметим, что при этом

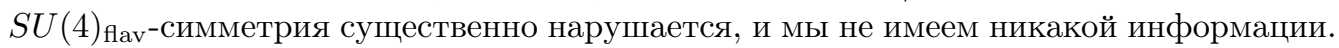




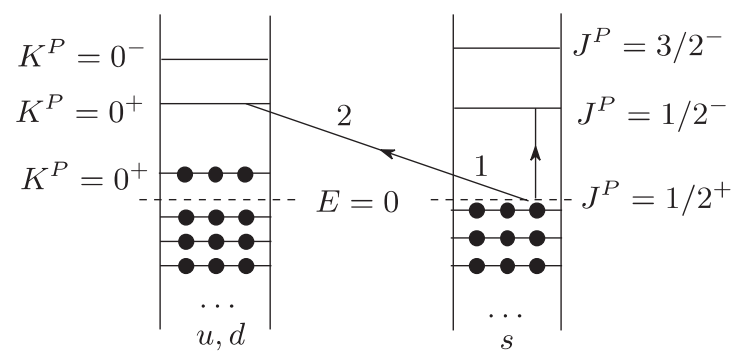

Рис. 4. Возможные переходы $s$-кварка с самого высокого заполненного уровня на возбужденный уровень $s$-кварка (переход 1) и на возбужденные уровни $u$-, $d$-кварков (переход 2 ).

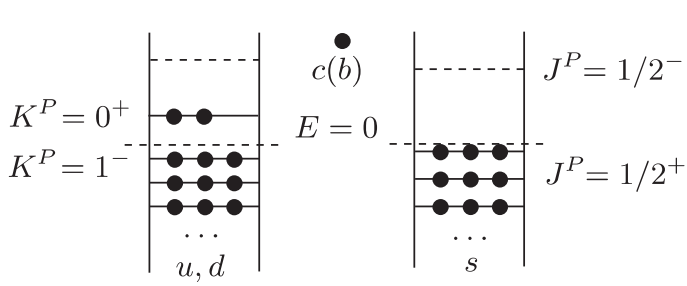

a

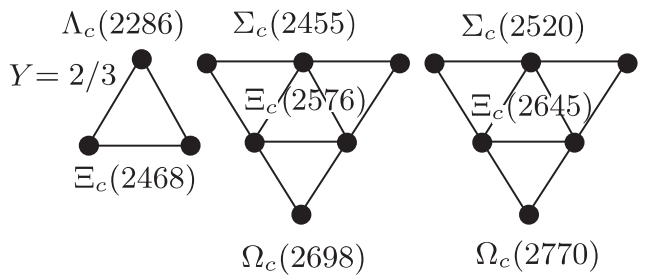

$\left(\overline{\mathbf{3}}, 1 / 2^{+}\right)$

$\left(6,1 / 2^{+}\right)$

б

Рис. 5. Заполнение $u-, d$ - и $s$-оболочек для основного состояния очарованных барионов (а) и $S U(3)$-мультиплеты, порожденные такой схемой заполнения (б): $\left(\overline{\mathbf{3}}, 1 / 2^{+}\right),\left(\mathbf{6}, 1 / 2^{+}\right)$и $\left(\mathbf{6}, 3 / 2^{+}\right)$.

Заполнение дираковских уровней для основного состояния $c$ - (или $b$-) бариона показано на рис. 5 a: в $0^{+}$-оболочке для $u$-, $d$-кварков присутствует дырка, поскольку там имеется только $N_{\text {c }}-1$ кварков в антисимметричном по цвету состоянии. Добавление тяжелого кварка делает полное состояние "бесцветным".

Как и в случае легких барионов, схема заполнения сама по себе не определяет квантовые числа состояния: они возникают из квантования $S U(3)_{\text {flav }}$ и $S O(3)_{\text {space- }^{-}}$ вращений данной схемы заполнения. Сделаем это для основного состояния тяжелых барионов.

Прежде всего найдем гиперзаряд схемы заполнения: в этом случае он равен $\widetilde{Y}=\left(N_{\mathrm{c}}-1\right) / 3$, поскольку имеется $N_{\mathrm{c}}-1 u$-, $d$-кварков, каждый из которых имеет гиперзаряд, равный $1 / 3$. При $N_{\mathrm{c}}=3$ имеем $\widetilde{Y}=2 / 3$. Имеются два $S U(3)$-мультиплета, содержащие частицы с гиперзарядом $2 / 3$ : антитриплет $\overline{\mathbf{3}}(p=0, q=1)$ и секстет 6 $(p=2, q=0)$; таким образом, это разрешенные мультиплеты (см. рис. 5б). Чему же равны их спины?

В представлении $\overline{\mathbf{3}}$ есть одна частица с $\widetilde{Y}=2 / 3$, поэтому ее изоспин равен $\widetilde{T}=0$. Возможный спин мультиплета находится из закона сложения векторов:

$$
\mathbf{J}=\widetilde{\mathbf{T}}+\mathbf{J}_{\mathrm{h}}
$$


Поэтому единственный возможный спин антитриплета равен $1 / 2$, а четность положительная. Его вращательная энергия согласно (16) равна

$$
E_{\text {rot }}^{(\overline{\mathbf{3}})}=\frac{1}{2 I_{2}}
$$

(более подробный вывод выражения для вращательной энергии в этом случае можно найти в работе [4]).

В представлении 6 имеются три частицы с $\widetilde{Y}=2 / 3$, поэтому их изоспин равен $\widetilde{T}=1$. Тогда из (19) находим, что имеется два секстета, один со спином $1 / 2$, а другой со спином $3 / 2$. Они вырожденны в главном порядке:

$$
E_{\mathrm{rot}}^{(\mathbf{6})}=\frac{1}{2 I_{2}}+\frac{1}{I_{1}}
$$

Таким образом, из схемы заполнения, приведенной на рис. 5а, следуют три $S U(3)-$ мультиплета (см. рис. 56$):\left(\overline{\mathbf{3}}, 1 / 2^{+}\right),\left(\mathbf{6}, 1 / 2^{+}\right)$и $\left(\mathbf{6}, 3 / 2^{+}\right)$. Два последних являются вырожденными (однако вырождение снимается в следующем, $1 / N_{\mathrm{c}}^{2}$, порядке, а также за счет $1 / m_{\mathrm{h}}$-поправок), в то время как центр антитриплета отделен от центра секстета вращательной энергией $\Delta E_{\mathrm{rot}}=1 / I_{1}$. Расщепление внутри мультиплетов, претерпевающих явное нарушение $S U(3)$-симметрии массой странного кварка, равно $O\left(m_{s} N_{\mathrm{c}}\right)$. Если $m_{s}$ интерпретируется как малое возмущение, $m_{s}=O\left(1 / N_{\mathrm{c}}^{2}\right)$, как мы и утверждали [3], то расщепление внутри секстета должно быть эквидистантно с хорошей точностью. Сопоставим эти предсказания с существующими на настоящий момент данными.

Имеются подходящие кандидаты для описанных выше мультиплетов в основном состоянии: $\Lambda_{c}(2286)$ и $\Xi_{c}(2468)$ для $\left(\overline{\mathbf{3}}, 1 / 2^{+}\right) ; \Sigma_{c}(2455), \Xi_{c}(2576)$ и $\Omega_{c}(2698)$ для $\left(6,1 / 2^{+}\right)$; наконец, $\Sigma_{c}(2520), \Xi_{c}(2645)$ и $\Omega_{c}(2770)$ предположительно образуют мультиплет $\left(6,3 / 2^{+}\right)$(см. рис. 5б). Строго говоря, квантовые числа $J^{P}$ большинства этих барионов непосредственно не измеряются, возможно, они отличаются от указанных. В предположении, что они правильны, наблюдаемые очарованные барионы с положительной четностью образуют в точности те мультиплеты, которые следуют из коллективного квантования.

Расщепление внутри двух секстетов эквидистантны с высокой точностью, это подтверждает, что $m_{s}$ можно интерпретировать как малое возмущение. Если величина $m_{s}$ не мала, то должны быть существенные $O\left(m_{s}^{2}\right)$-поправки к массам, которые должны нарушать эквидистантный характер спектра секстета.

Центры трех мультиплетов следующие:

$$
\begin{aligned}
& m\left(\overline{\mathbf{3}}, 1 / 2^{+}\right)=\frac{2287+2 \cdot 2468}{3}=2408 \mathrm{M \ni B}, \\
& m\left(\mathbf{6}, 1 / 2^{+}\right)=\frac{3 \cdot 2455+2 \cdot 2576+2698}{6}=2536 \mathrm{M \ni B} \\
& m\left(\mathbf{6}, 3 / 2^{+}\right)=\frac{3 \cdot 2520+2 \cdot 2645+2770}{6}=2603 \mathrm{M \ni B} .
\end{aligned}
$$




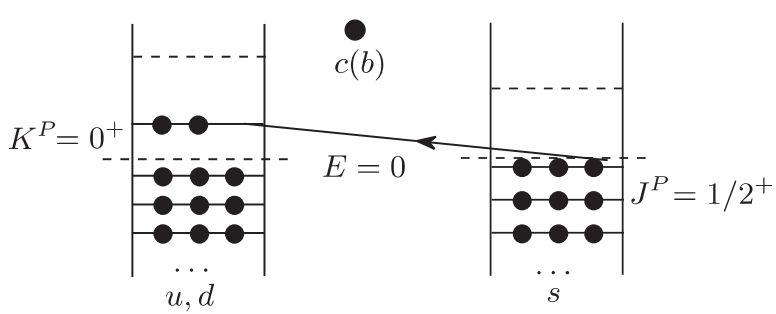

a

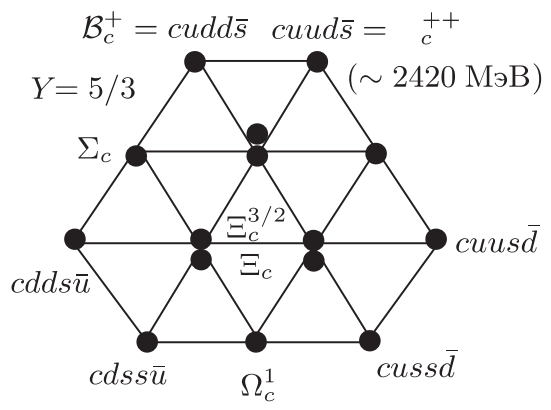

б

Рис. 6. Стрелка показывает возможное возбуждение Гамова-Теллера (а), приводящее к очарованным бета-пентакваркам $\mathcal{B}_{c}^{++}=c u u d \bar{s}, \mathcal{B}_{c}^{+}=c u d d \bar{s}$, в действительности принадлежащим $S U(3)$-мультиплету $\overline{\mathbf{1 5}}$ с положительной четностью (б).

Хотя два секстета не являются точно вырожденными, их расщепление 67 МэВ (неучтенный $1 / N_{\mathrm{c}}^{2}$-эффект) гораздо меньше, чем расщепление между антитриплетом и средней массой секстетов, равное

$$
\frac{2536+2603}{2}-2408=162 \mathrm{M \ni B}=E_{\text {rot }}^{(\mathbf{6})}-E_{\text {rot }}^{(\overline{\mathbf{3}})}=\frac{1}{I_{1}}=O\left(\frac{1}{N_{\mathrm{c}}}\right) .
$$

Более того, эта величина должна быть сравнима с моментом инерции, следующим из расщепления между легкими барионами, $\left(\mathbf{1 0}, 3 / 2^{+}\right)$и $\left(\mathbf{8}, 1 / 2^{+}\right)$, что приводит к значению $1 / I_{1}=155 \mathrm{MэB} \mathrm{(см.} \mathrm{раздел} \mathrm{6).} \mathrm{Близость} \mathrm{двух} \mathrm{совершенно} \mathrm{различных}$ определений момента инерции подтверждает основную идею о том, что разумно рассматривать как легкие, так и тяжелые барионы с одинаковой точки зрения при больших $N_{\mathrm{c}}{ }^{2)}$.

\section{9. НОВЫЕ ЭКЗОТИЧЕСКИЕ И УСТОЙЧИВЫЕ ОЧАРОВАННЫЕ И $b$-БАРИОНЫ}

Как было отмечено в работе [4], дырка в оболочке основного состояния $0^{+}$(см. рис. 5) достаточно провокационна: ее можно заполнить или за счет возбуждения $u$-, $d$-кварка из лежащих ниже оболочек, или за счет недиагонального перехода Гамова-Теллера из самой высокой заполненной оболочки $s$-кварка. Во втором случае соответствующий резонанс очарованных барионов будет экзотическим пентакварком (см. рис. 6).

Массы "бета-барионов" $\mathcal{B}_{c}^{++}=c u u d \bar{s}, \mathcal{B}_{c}^{+}=c u d d \bar{s}$ и $\mathcal{B}_{b}^{+}=b u u d \bar{s}, \mathcal{B}_{b}^{0}=b u d d \bar{s}$ непосредственно зависят от положения самого высокого заполненного уровня $s$-кварков по отношению к уровню основного состояния $u$-, $d$-кварков. В работе [4] аргументированно утверждалось, что эта разность в энергии может оказаться весьма малой, 100-200 МэВ, так что экзотические бета-барионы окажутся легкими,

${ }^{2)}$ Соотношение $m(\mathbf{6})-m(\overline{\mathbf{3}})=(2 / 3)(m(\Delta)-m(N))$ впервые было получено в работе [19] путем применения модели Скирма к тяжелым барионам - подход, близкий по духу применяемому в настоящей работе. 
$m\left(\mathcal{B}_{c}\right) \approx 2420$ МэВ, а потому и устойчивыми по отношению к распадам по сильному взаимодействию ${ }^{3)}$. Существует возможность их наблюдения на Большом адронном коллайдере и $b$-фабриках.

\section{0. ЗАКЛЮЧЕНИЕ}

Если число цветов $N_{\text {c }}$ рассматривается как свободный алгебраический параметр, то барионные резонансы классифицируются просто. При больших $N_{\text {c }}$ все барионные резонансы в основном определяются внутренним кварковым спектром, который принимает некоторую предельную форму при $N_{\mathrm{c}} \rightarrow \infty$. Этот спектр один и тот же и для легких барионов $q \ldots q q$ с $N_{\text {с }}$ легкими кварками $q$, и для тяжелых барионов $q \ldots q Q$ с $N_{\mathrm{c}}-1$ легкими кварками и одним тяжелым кварком $Q$, поскольку различие является эффектом порядка $1 / N_{\text {c }}$.

Различными способами можно возбудить кварковые уровни, называемые одночастичными возбуждениями или возбуждениями частица-дырка; в обоих случаях энергия возбуждения есть величина порядка $O(1)$. Помимо каждого однокваркового или кварк-антикваркового возбуждения в общем случае имеется зона $S U(3)$-мультиплетов барионных резонансов, которые представляют собой вращательные состояния бариона как целого. Поэтому расщепление между мультиплетами есть величина порядка $O\left(1 / N_{\mathrm{c}}\right)$. Вращательная зона завершается, когда энергия вращения достигает $O(1)$.

В действительности $N_{\mathrm{c}}=3$, и описанная выше идеализированная иерархия масштабов несколько размыта. Тем не менее изучение спектра барионных резонансов выявляет определенную иерархию, которую можно схематически представить следующим образом:

- масса бариона: $O\left(N_{\mathrm{c}}\right)$ (характерная величина - средняя масса октета основного состояния $1200 \mathrm{MэB);}$

- однокварковое возбуждение и возбуждение частица-дырка во внутреннем спектре: $O(1)$ (характерная величина - возбуждение резонанса Ропера $400 \mathrm{MэB);}$

- расщепление между центрами $S U(3)$-мультиплетов, возникающее из-за вращательных возбуждений данного внутреннего состояния: $O\left(1 / N_{\mathrm{c}}\right)$ (характерная величина 133 МэВ);

- расщепление между центрами вращательных мультиплетов, различающихся спинами, т. е. вырожденных в главном порядке: $O\left(1 / N_{\mathrm{c}}^{2}\right)$ (характерная величина $44 \mathrm{M \ni B);}$

- расщепление внутри данного мультиплета вследствие ненулевой массы странного кварка: $O\left(m_{s} N_{\mathrm{c}}\right)$ (характерная величина $140 \mathrm{MэB).}$

Практически мы показали, что все барионные резонансы вплоть до 2 ГэВ, составленные из легких кварков, можно понимать как вращательные возбуждения вокруг некоторых переходов между внутренними кварковыми уровнями. Квантовые числа резонансов и расщепления между мультиплетами из одной и той же вращательной зоны диктуются квантовыми числами внутренних кварковых уровней и, по-видимому, находятся в хорошем согласии с экспериментальными данными.

3) См. работу [4], где рассматривался новоявленный антидекапентаплет экзотических очарованных пентакварков (или $b$-пентакварков). 
Содержание и расщепление низших очарованных (и $b$-кварков) барионных мультиплетов также находятся в согласии с их интерпретацией как вращательной зоны вблизи схемы заполнения основного состояния.

В настоящей работе мы сконцентрировались на алгебраических аспектах проблемы, оставив в стороне динамические аспекты. Динамические модели должны ответить на вопрос о том, почему внутренние кварковые уровни для $u$-, $d$-кварков с $K^{P}=0^{ \pm}, 1^{ \pm}, 2^{ \pm}$и уровни $s$-кварков с $J^{P}=1 / 2^{ \pm}, 3 / 2^{ \pm}$и т. д. обладают конкретными энергиями, приведенными в таблице в п. 6.3. Однако нам кажется, что это все же шаг вперед: вместо необходимости объяснения двух сотен резонансов требуется объяснить положения только нескольких внутренних кварковых уровней. Рис. 1 иллюстрирует, что требуемый внутренний спектр можно получить исходя из разумного набора средних полей.

Предлагаемая схема интерпретации барионных резонансов имеет многочисленные феноменологические следствия, которые можно изучать даже до того, как будет рассмотрена настоящая динамика. А именно, из того факта, что определенные группы $S U(3)$-мультиплетов принадлежат одной и той же вращательной зоне, связанной с одним и тем же однокварковым переходом, следуют соотношения между их взаимодействиями, формфакторами, расщеплениями внутри мультиплетов из-за ненулевого значения $m_{s}$ и т. д.

Благодарности. Мы благодарны К. Геке и М. Полякову за полезные обсуждения. Работа Д. И. Дьяконова и В. Ю. Петрова выполнена при финансовой поддержке Deutsche Forschungsgemeinschaft (DFG), а также РФФИ (грант № 09-02-01198) и Программы поддержки ведущих научных школ (грант НШ-3628.2008.2). Работа А. А. Владимирова поддержана SFB/TR16.

\section{Список литературы}

[1] E. Witten, Nucl. Phys. B, 160:1 (1979), 57-115.

[2] D. I. Diakonov, V. Yu. Petrov, The Multifaceted Skyrmion, eds. G. Brown, M. Rho, World Scientific, Hackensack, NJ, 2010, arXiv: 0812.1212.

[3] D. I. Diakonov, Писъма в ЖЭТФ, 90:6 (2009), 451-455, arXiv: 0812.3418; Nucl. Phys. A, 827:1-4 (2009), 264c-266c, arXiv: 0901.1373.

[4] D. I. Diakonov, Chinese Phys. C, 34:9 (2010), 1298-1302, arXiv: 0912.3175; Prediction of new charmed and bottom exotic pentaquarks, arXiv: 1003.2157.

[5] D. I. Diakonov, V. Yu. Petrov, Spontaneous breaking of chiral symmetry in the instanton vacuum, Препринт ЛИЯФ-1153, ЛИЯФ, Л., 1986; Адронная материя в экстремальных условиях, ред. Г. М. Зиновьев, В. П. Шелест, Наукова думка, Киев, 1986, 192-225; D. I. Diakonov, Progr. Part. Nucl. Phys., 51:1 (2003), 173-222, arXiv: hep-ph/0212026.

[6] Д. И. Дьяконов, В. Ю. Петров, Писъма в ЖЭТФ, 43:2 (1986), 57-59.

[7] A. Hosaka, H. Toki, Phys. Rep., 277:2-3 (1996), 65-188; A. Hosaka, Phys. Lett. B, 571:1-2 (2003), 55-60.

[8] A. Blotz, D. I. Diakonov, K. Goeke, N. W. Park, V. Yu. Petrov, P. V. Pobylitsa, Nucl. Phys. A, 555:4 (1993), 765-792.

[9] W. Pauli, S. M. Dancoff, Phys. Rev., 62:3-4 (1942), 85-108.

[10] T. H. R. Skyrme, Proc. Roy. Soc. Lond. A, 260:1300 (1961), 127-138; Nucl. Phys., 31:3-4 (1962), 556-569. 
[11] D. I. Diakonov, V. Yu. Petrov, P.V. Pobylitsa, Nucl. Phys. B, 306:4 (1988), 809-848; D. I. Diakonov, Eur. Phys. J. A, 24, Suppl. 1:1 (2005), 3-8; Chiral symmetry and pentaquarks, arXiv: hep-ph/0412272.

[12] E. Witten, Nucl. Phys. B, 223:2 (1983), 433-444.

[13] V. Guzey, M. V. Polyakov, Ann. Phys., 13:11-12 (2004), 673-681, arXiv: hep-ph/0512355.

[14] J. P. Elliot, T. H. R. Skyrme, Proc. Roy. Soc. Lond. A, 232:1191 (1955), 561-566.

[15] L. Ya. Glozman, D. O. Riska, Phys. Rep., 268:4 (1996), 263-303, arXiv: hep-ph/9505422.

[16] P. V. Pobylitsa, Phys. Rev. D, 69:7 (2004), 074030, 19 pp., arXiv: hep-ph/0310221.

[17] B.S. Zou, Eur. Phys. J. A, 35:3 (2008), 325-328, arXiv: 0711.4860.

[18] D. I. Diakonov, V. Yu. Petrov, M. V. Polyakov, Z. Phys. A, 359:3 (1997), 305-314, arXiv: hep-ph/9703373.

[19] A. Momen, J. Schechter, A. Subbaraman, Phys. Rev. D, 49:11 (1994), 5970-5978, arXiv: hep-ph/9401209. 